\title{
TG19
}

\section{Formation Evaluation in Tight Gas Reservoirs}

\section{S.M. Hansen* (Schlumberger)}

\section{SUMMARY}

Tight gas reservoirs have been defined as any reservoir having permeability less than $0.1 \mathrm{mD}$ or a reservoir that will not produce commercially without stimulation. A tight gas reservoir is more difficult to evaluate. In order to completely evaluate and characterize these reservoirs to enable a successful and commercial development a more extensive set of data is required. Formation evaluation of these reservoirs consists of five components: lithology(mineralogy), texture, depositional environment, present day stress, and tectonic history

(fracture types and orientation). The successful integration of these geological, petrophysical, and geo-mechanical disciplines is necessary in order to evaluate these reservoirs. 
Tight gas reservoirs have been defined as any reservoir having permeability less than $0.1 \mathrm{mD}$ or a reservoir that will not produce commercially without stimulation. A tight gas reservoir is more difficult to evaluate. In order to completely evaluate and characterize these reservoirs to enable a successful and commercial development a more extensive set of data is required. Formation evaluation of these reservoirs consists of five components: lithology (mineralogy), texture, depositional environment, present day stress, and tectonic history (fracture types and orientation). The successful integration of these geological, petrophysical, and geo-mechanical disciplines is necessary in order to evaluate these reservoirs.

The mineralogical composition of tight sandstone reservoirs may be quite variable. Even though mineralogical complexity is usually a sign of textural complexity, tight sandstones may also have a very simple mineralogy, but exhibit a complex texture and a corresponding low permeability.
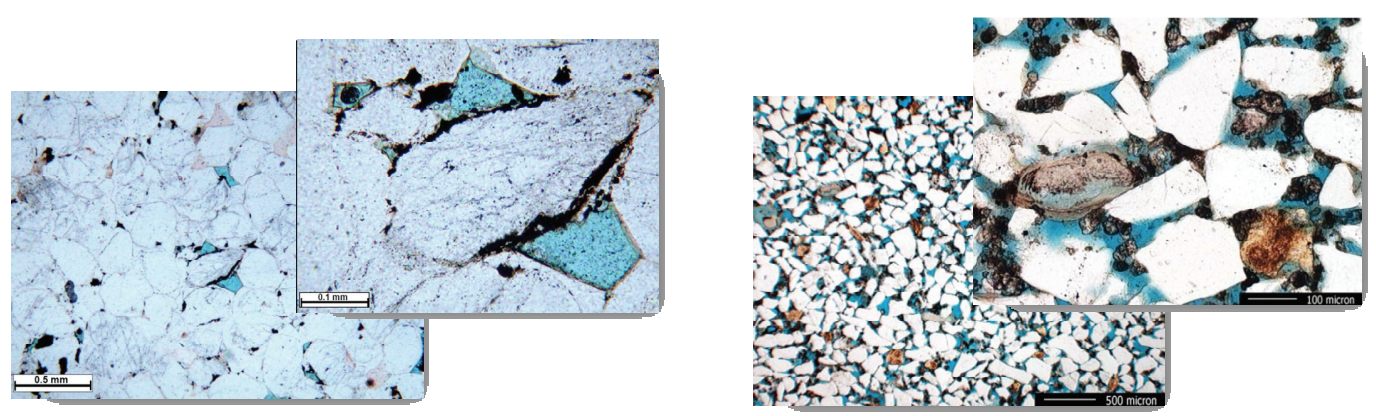

Figure 1 Two tight sandstone reservoirs with similarly low permeability $(<1 \mathrm{mD})$ exhibit contrasting mineralogy and texture. Left - Quartzite (98\% quartz grains \& overgrowth) with $\rho_{m a}=2.65 \mathrm{~g} / \mathrm{cc}$ and $R t=500 \Omega . m ;$ Right - Chlorite-lined quartz with $\rho_{m a}=2.82 \mathrm{~g} / \mathrm{cc}$ and $R t=2 \Omega . m$.

Fractures in tight gas reservoirs, both natural and artificially induced, are the principal contributor to production. Fractures detected within the wellbore need to be classified into either induced by the drilling process, or naturally occurring fractures. The natural fractures are further classified into distinct sets based on orientation and type (lithology bound, continuous, healed, partially healed or brecciated). The fracture density and length can be measured in all wells, whereas fracture aperture and porosity (and at times fracture permeability) of each individual fracture set can be calculated when the well is drilled with a water-based mud.

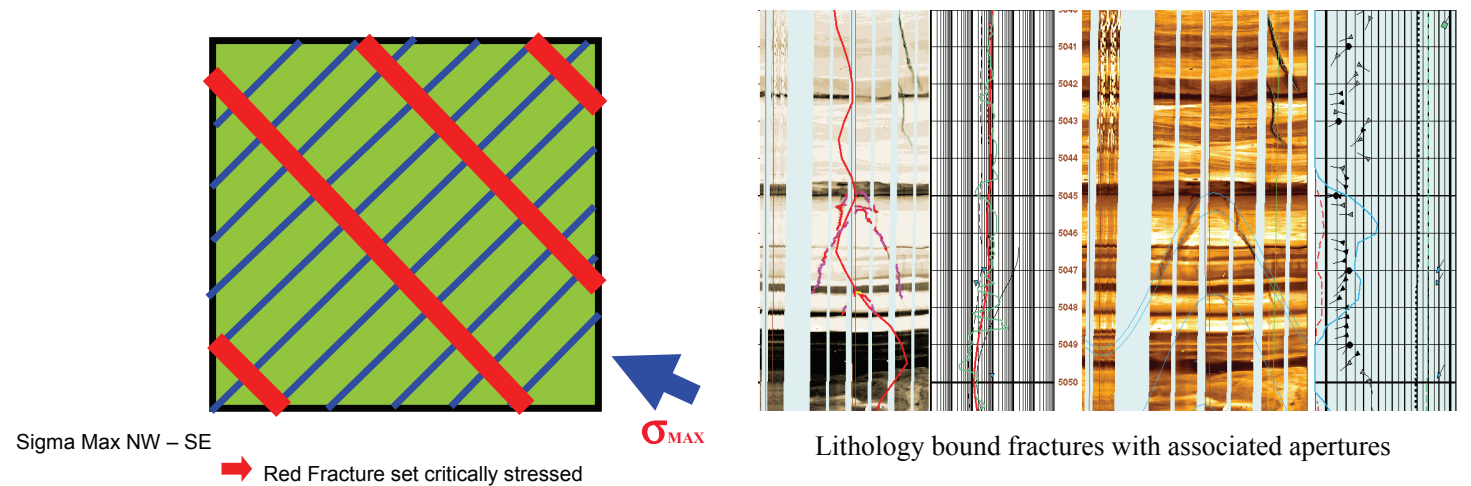

Figure 2 Determination of critically stressed fractures and fracture types.

Next to fractures, textural variations provide the second largest contribution to production. These textural variations within the reservoir may or may not be related to their mineralogy. When the textural variations are not caused by mineral variations, detailed lithological analysis will not be sufficient; a textural log is required. Textural logs may be borehole micro-resistivity images or NMR pore-size distribution. The types of textural variations and detection algorithms vary with lithology. In carbonates the primary control is the amount and connectivity of secondary (vuggy) porosity. In 
clastics the primary textural variation is correlated to grain size and the degree of grain sorting. Both of these variations are measurable with high-resolution borehole images.
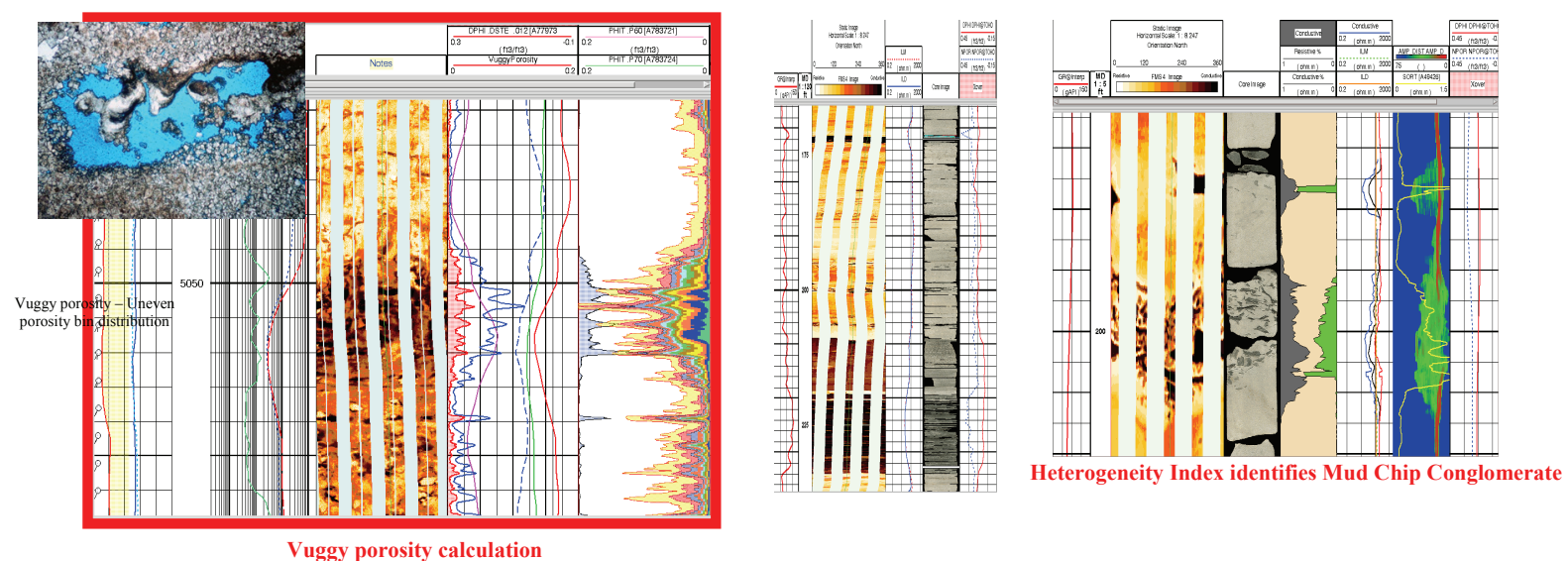

Figure 3 Textural computations in carbonates on left and clastics on right.

NMR (Nuclear Magnetic Resonance) logs can also measure textural properties of a tight reservoir, regardless of its mineral composition. The pore size distribution from a NMR log is used to estimate the capillary-bound water volume, the rock permeability, and even a pseudo-capillary pressure profile that can be calibrated into a continuous water saturation profile, independent of formation resistivity and electrical (Archie) parameters.

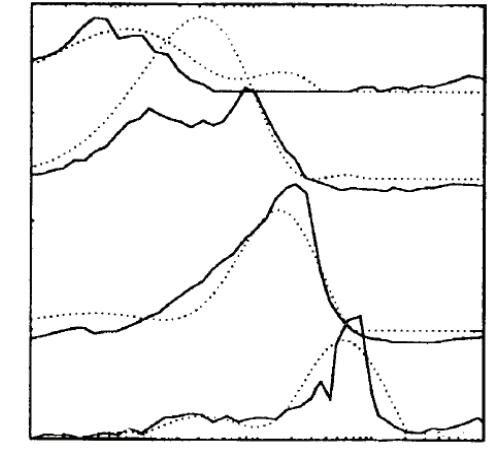

$\log _{10}$ time constant, sec

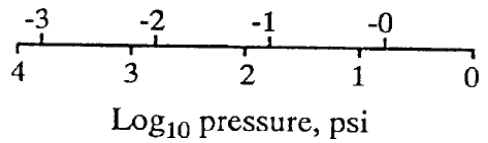

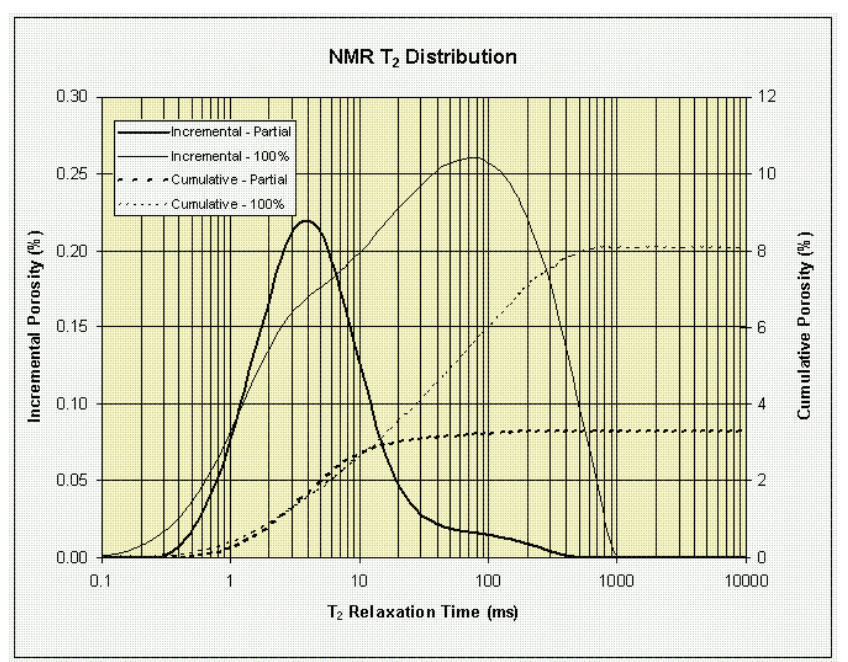

Figure 4 Left: NMR T2 distribution (dotted) and Hg injection pressure profile (continuous) offer similar information about rock texture (Ref. Straley et al, 1997). Right: In a low-porosity (8 pu) and low-permeability $(0.83 \mathrm{mD})$ sandstone, the NMR T2 profile (before and after centrifuge) clearly describes the pore size distribution, within the range of downhole NMR tools (0.3 to $3000 \mathrm{~ms}$ ) (Ref. Worldwide Core Catalog).

Borehole images and/or sonic measurements can determine the present day borehole stresses acting on the wellbore. Ultilizing borehole images allows one to determine the type of borehole deformation taking place due to the stress. The deformation of the wellbore is determined by the variations in the tangential, axial and radial stress acting on the wellbore, are classified into wide breakout, narrow breakout, high angle echelon fractures, and tensile failure fractures. The type of deformation in the wellbore will establish the present day stress orientations which will discern which one of these fracture sets are critically stressed, and thus most likely to contribute to production. 


\section{EAGE}

When designing a stimulation job, depositional environment in clastic environments is an extremely important component. Knowledge of the facies type (channel, bar, etc) and its orientation allows the proper stimulation treatment to be designed so that a fracture will not extend laterally out of the reservoir.

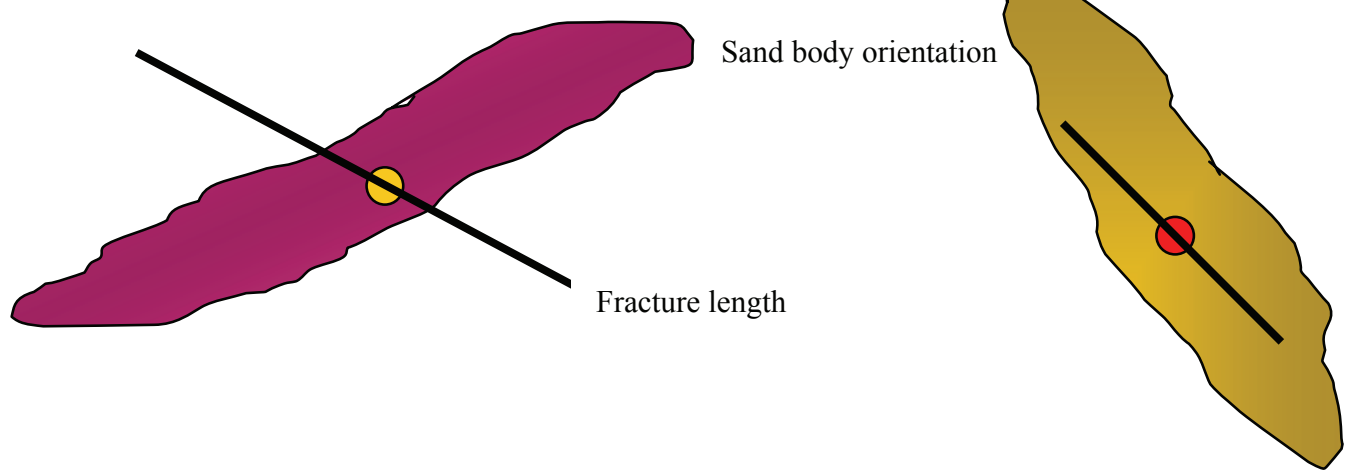

Figure 5 Depositional environment and facies orientation in conjunction with present day principal stress direction influencing stimulation length design. 